Acta Math., 219 (2017), 1-16

DOI: 10.4310/ACTA.2017.v219.n1.a1

(C) 2017 by Institut Mittag-Leffler. All rights reserved

\title{
Asymptotic behavior of flows by powers of the Gaussian curvature
}

by

\author{
Simon BREndLE \\ Columbia University \\ New York, NY, U.S.A.
}

\author{
Kyeongsu Choi \\ Columbia University \\ New York, NY, U.S.A
}

Panagiota Daskalopoulos

\author{
Columbia University \\ New York, NY, U.S.A.
}

\section{Introduction}

Parabolic flows for hypersurfaces play an important role in differential geometry. One fundamental example is the flow by mean curvature (see [18]). In this paper, we consider flows where the speed is given by some power of the Gaussian curvature. More precisely, given an integer $n \geqslant 2$ and a real number $\alpha>0$, a 1-parameter family of immersions $F: M^{n} \times[0, T) \rightarrow \mathbb{R}^{n+1}$ is a solution of the $\alpha$-Gauss curvature flow if, for each $t \in[0, T)$, $F\left(M^{n}, t\right)=\Sigma_{t}$ is a complete convex hypersurface in $\mathbb{R}^{n+1}$ and $F(\cdot, t)$ satisfies

$$
\frac{\partial}{\partial t} F(p, t)=-K^{\alpha}(p, t) \nu(p, t) .
$$

Here, $K(p, t)$ and $\nu(p, t)$ are the Gauss curvature and the outward-pointing unit normal vector of $\Sigma_{t}$ at the point $F(p, t)$, respectively.

THeOREM 1. Let $\Sigma_{t}$ be a family of closed, strictly convex hypersurfaces in $\mathbb{R}^{n+1}$ moving with speed $-K^{\alpha} \nu$, where $\alpha \geqslant 1 /(n+2)$. Then, either the hypersurfaces $\Sigma_{t}$ converge to a round sphere after rescaling, or we have $\alpha=1 /(n+2)$ and the hypersurfaces $\Sigma_{t}$ converge to an ellipsoid after rescaling.

Flows by powers of the Gaussian curvature have been studied by many authors, starting with the seminal paper of Firey [14] in 1974. Tso [21] showed that the flow

The first-named author was supported in part by the National Science Foundation under grant DMS-1649174. The third-named author was supported in part by the National Science Foundation under grant DMS-1600658. 
exists up to some maximal time, when the enclosed volume converges to zero. In the special case $\alpha=1 / n$, Chow [9] proved convergence to a round sphere. Moreover, Chow [10] obtained interesting Harnack inequalitites for flows, by powers of the Gaussian curvature (see also [17]). In the affine invariant case $\alpha=1 /(n+2)$, Andrews [1] showed that the flow converges to an ellipsoid. This result can alternatively be derived from a theorem of Calabi [7], which asserts that the only self-similar solutions for $\alpha=1 /(n+2)$ are ellipsoids. The arguments in [7] and [1] rely crucially on the affine invariance of the equation, and do not generalize to other exponents. In the special case of surfaces in $\mathbb{R}^{3}(n=2)$, Andrews [2] proved that flow converges to a sphere when $\alpha=1$; this was later extended in [4] to the case $n=2$ and $\alpha \in\left[\frac{1}{2}, 2\right]$. The results in [2] and [4] rely on an application of the maximum principle to a suitably chosen function of the curvature eigenvalues; these techniques do not appear to work in higher dimensions. However, it is known that the flow converges to a self-similar solution for every $n \geqslant 2$ and every $\alpha \geqslant 1 /(n+2)$. This was proved by Andrews [3] for $\alpha \in[1 /(n+2), 1 / n]$; by Guan and Ni [16] for $\alpha=1$; and by Andrews, Guan, and $\mathrm{Ni}[5]$ for all $\alpha \in(1 /(n+2), \infty)$. One of the key ingredients in these results is a monotonicity formula for an entropy functional. This monotonicity was discovered by Firey [14] in the special case $\alpha=1$.

Thus, the problem can be reduced to the classification of self-similar solutions. In the affine invariant case $\alpha=1 /(n+2)$, the self-similar solutions were already classified by Calabi [7]. In the special case when $\alpha \geqslant 1$ and the hypersurfaces are invariant under antipodal reflection, it was shown in [5] that the only self-similar solutions are round spheres. Very recently, the case $1 / n \leqslant \alpha<1+1 / n$ was solved in [8] as part of K. Choi's $\mathrm{Ph}$. D. thesis. In particular, this includes the case $\alpha=1$ conjectured by Firey [14].

Finally, we note that there is a substantial literature on other fully non-linear parabolic flows for hypersurfaces (see e.g. [13], [6], [20]) and for Riemannian metrics (cf. [12]). In particular, Gerhardt [15] studied convex hypersurfaces moving outward with speed $K^{\alpha} \nu$, where $\alpha<0$. Note that, for $\alpha<0$, one can show using the method of moving planes that any convex hypersurface satisfying $K^{\alpha}=\langle x, \nu\rangle$, where $\alpha<0$, is a round sphere. Using an a-priori estimate in [11], Gerhardt [15] proved that the flow converges to a round sphere after rescaling.

We now give an outline of the proof of Theorem 1. In view of the discussion above, it suffices to classify all closed self-similar solutions to the flow. The self-similar solutions $\Sigma=F\left(M^{n}\right)$ satisfy the equation

$$
K^{\alpha}=\langle F, \nu\rangle .
$$

To classify the solutions of $\left(*_{\alpha}\right)$, we distinguish two cases. 
First, suppose that $\alpha \in\left[1 /(n+2), \frac{1}{2}\right]$. In this case, we consider the quantity

$$
Z=K^{\alpha} \operatorname{tr}(b)-\frac{n \alpha-1}{2 \alpha}|F|^{2},
$$

where $b$ denotes the inverse of the second fundamental form. The motivation for the quantity $Z$ is that $Z$ is constant when $\alpha=1 /(n+2)$ and $\Sigma$ is an ellipsoid. Indeed, if $\alpha=1 /(n+2)$ and $\Sigma=\left\{x \in \mathbb{R}^{n+1}:\langle S x, x\rangle=1\right\}$ for some positive definite matrix $S$ with determinant 1 , then $K^{1 /(n+2)}=\langle F, \nu\rangle$ and

$$
Z=K^{1 /(n+2)} \operatorname{tr}(b)+|F|^{2}=\operatorname{tr}\left(S^{-1}\right) .
$$

Hence, in this case $Z$ is constant, and equals the sum of the squares of the semi-axes of the ellipsoid.

Suppose now that $\Sigma=F\left(M^{n}\right)$ is a solution of $\left(*_{\alpha}\right)$ for some $\alpha \in\left[1 /(n+2), \frac{1}{2}\right]$. We show that $Z$ satisfies an inequality of the form

$$
\alpha K^{\alpha} b^{i j} \nabla_{i} \nabla_{j} Z+(2 \alpha-1) b^{i j} \nabla_{i} K^{\alpha} \nabla_{j} Z \geqslant 0 .
$$

The strong maximum principle then implies that $Z$ is constant. By examining the case of equality, we are able to show that either $\nabla h=0$, or $\alpha=1 /(n+2)$ and the cubic form vanishes. This shows that either $\Sigma$ is a round sphere, or $\alpha=1 /(n+2)$ and $\Sigma$ is an ellipsoid.

Finally, we consider the case $\alpha \in\left(\frac{1}{2}, \infty\right)$. As in [8], we consider the quantity

$$
W=K^{\alpha} \lambda_{1}^{-1}-\frac{n \alpha-1}{2 n \alpha}|F|^{2} .
$$

By applying the maximum principle, we can show that any point where $W$ attains its maximum is umbilical. From this, we deduce that any maximum point of $W$ is also a maximum point of $Z$. Applying the strong maximum principle to $Z$, we are able to show that $Z$ and $W$ are both constant. This implies that $\Sigma$ is a round sphere.

\section{Preliminaries}

We first recall the notation:

- The metric is given by $g_{i j}=\left\langle F_{i}, F_{j}\right\rangle$, where $F_{i}:=\nabla_{i} F$. Moreover, $g^{i j}$ denotes the inverse of $g_{i j}$, so that $g^{i j} g_{j k}=\delta_{k}^{i}$. Also, we use the notation $F^{i}=g^{i j} F_{j}$.

- We denote by $H$ and $h_{i j}$ the mean curvature and second fundamental form, respectively.

- For a strictly convex hypersurface, we denote by $b^{i j}$ the inverse of the second fundamental form $h_{i j}$, so that $b^{i j} h_{j k}=\delta_{k}^{i}$. Moreover, $\operatorname{tr}(b)$ will denote the trace of $b$, i.e. the reciprocal of the harmonic mean curvature. 
- We denote by $\mathcal{L}$ the operator $\mathcal{L}=\alpha K^{\alpha} b^{i j} \nabla_{i} \nabla_{j}$.

- We denote by $C_{i j k}$ the cubic form

$$
C_{i j k}=\frac{1}{2} K^{-1(n+2)} \nabla_{k} h_{i j}+\frac{1}{2} h_{j k} \nabla_{i} K^{-1 /(n+2)}+\frac{1}{2} h_{k i} \nabla_{j} K^{-1 /(n+2)}+\frac{1}{2} h_{i j} \nabla_{k} K^{-1 /(n+2)} .
$$

We next derive some basic equations.

Proposition 2. Given a strictly convex smooth solution $F: M^{n} \rightarrow \mathbb{R}^{n+1}$ of $\left(*_{\alpha}\right)$, the following equations hold:

$$
\begin{aligned}
\nabla_{i} b^{j k}= & -b^{j l} b^{k m} \nabla_{i} h_{l m}, \\
\mathcal{L}|F|^{2}= & 2 \alpha K^{\alpha} b^{i j}\left(g_{i j}-h_{i j} K^{\alpha}\right)=2 \alpha K^{\alpha} \operatorname{tr}(b)-2 n \alpha K^{2 \alpha}, \\
\nabla_{i} K^{\alpha}= & h_{i j}\left\langle F, F^{j}\right\rangle, \\
\mathcal{L} K^{\alpha}= & \left\langle F, F_{i}\right\rangle \nabla_{i} K^{\alpha}+n \alpha K^{\alpha}-\alpha K^{2 \alpha} H, \\
\mathcal{L} h_{i j}=- & K^{-\alpha} \nabla_{i} K^{\alpha} \nabla_{j} K^{\alpha}+\alpha K^{\alpha} b^{p r} b^{q s} \nabla_{i} h_{r s} \nabla_{j} h_{p q} \\
& \quad\left\langle\left\langle F, F_{k}\right\rangle \nabla^{k} h_{i j}+h_{i j}+(n \alpha-1) h_{i k} h_{j}^{k} K^{\alpha}-\alpha K^{\alpha} H h_{i j},\right. \\
\mathcal{L} b^{p q}= & K^{-\alpha} b^{p r} b^{q s} \nabla_{r} K^{\alpha} \nabla_{s} K^{\alpha}+\alpha K^{\alpha} b^{p r} b^{q s} b^{i j} b^{k m} \nabla_{r} h_{i k} \nabla_{s} h_{j m} \\
& \quad+\left\langle F, F_{i}\right\rangle \nabla^{i} b^{p q}-b^{p q}-(n \alpha-1) g^{p q} K^{\alpha}+\alpha K^{\alpha} H b^{p q} .
\end{aligned}
$$

Proof. The relation $\nabla_{i}\left(b^{j k} h_{k l}\right)=\nabla_{i} \delta_{l}^{j}=0$ gives $h_{k l} \nabla_{i} b^{j k}=-b^{j k} \nabla_{i} h_{k l}$. This directly implies (1). We next compute

$$
\nabla_{i} \nabla_{j}|F|^{2}=2\left\langle\nabla_{i} F, \nabla_{j} F\right\rangle+2\left\langle F, \nabla_{i} \nabla_{j} F\right\rangle=2 g_{i j}-2 h_{i j}\langle F, \nu\rangle=2 g_{i j}-2 K^{\alpha} h_{i j},
$$

and hence

$$
\mathcal{L}|F|^{2}=2 \alpha K^{\alpha} \operatorname{tr}(b)-2 n \alpha K^{2 \alpha}
$$

This proves (2).

To derive equation (3), we differentiate $\left(*_{\alpha}\right)$ :

$$
\nabla_{i} K^{\alpha}=h_{i k}\left\langle F, F^{k}\right\rangle
$$

If we differentiate this equation again, we obtain

$$
\nabla_{i} \nabla_{j} K^{\alpha}=\left\langle F, F^{k}\right\rangle \nabla_{i} h_{j k}+h_{i j}-h_{i k} h_{j}^{k}\langle F, \nu\rangle=\left\langle F, F^{k}\right\rangle \nabla_{k} h_{i j}+h_{i j}-K^{\alpha} h_{i k} h_{j}^{k},
$$

and hence

$$
\mathcal{L} K^{\alpha}=\left\langle F, F^{k}\right\rangle \nabla_{k} K^{\alpha}+n \alpha K^{\alpha}-\alpha K^{2 \alpha} H .
$$


On the other hand, using (1) we compute

$$
\begin{aligned}
\nabla_{i} \nabla_{j} K^{\alpha} & =\nabla_{i}\left(\alpha K^{\alpha} b^{p q} \nabla_{j} h_{p q}\right) \\
& =\alpha K^{\alpha} b^{p q} \nabla_{i} \nabla_{j} h_{p q}+\alpha^{2} K^{\alpha} b^{r s} b^{p q} \nabla_{i} h_{r s} \nabla_{j} h_{p q}-\alpha K^{\alpha} b^{p r} b^{q s} \nabla_{i} h_{r s} \nabla_{j} h_{p q} .
\end{aligned}
$$

Using the commutator identity

$$
\begin{aligned}
\nabla_{i} \nabla_{j} h_{p q} & =\nabla_{i} \nabla_{p} h_{j q}=\nabla_{p} \nabla_{i} h_{j q}+R_{i p j m} h_{q}^{m}+R_{i p q m} h_{j}^{m} \\
& =\nabla_{p} \nabla_{q} h_{i j}+\left(h_{i j} h_{p m}-h_{i m} h_{j p}\right) h_{q}^{m}+\left(h_{i q} h_{p m}-h_{i m} h_{p q}\right) h_{j}^{m}
\end{aligned}
$$

we deduce that

$$
\begin{aligned}
\alpha K^{\alpha} b^{p q} \nabla_{i} \nabla_{j} h_{p q} & =\alpha K^{\alpha} b^{p q} \nabla_{p} \nabla_{q} h_{i j}+\alpha K^{\alpha} H h_{i j}-n \alpha K^{\alpha} h_{i m} h_{j}^{m} \\
& =\mathcal{L} h_{i j}+\alpha K^{\alpha} H h_{i j}-n \alpha K^{\alpha} h_{i m} h_{j}^{m} .
\end{aligned}
$$

Combining the equations above yields

$$
\begin{aligned}
\mathcal{L} h_{i j}=- & \alpha^{2} K^{\alpha} b^{r s} b^{p q} \nabla_{i} h_{r s} \nabla_{j} h_{p q}+\alpha K^{\alpha} b^{p r} b^{q s} \nabla_{i} h_{r s} \nabla_{j} h_{p q} \\
& +\left\langle F, F^{k}\right\rangle \nabla_{k} h_{i j}+h_{i j}+(n \alpha-1) h_{i k} h_{j}^{k} K^{\alpha}-\alpha K^{\alpha} H h_{i j} .
\end{aligned}
$$

This completes the proof of (5).

Finally, using (1), we obtain

$$
\begin{aligned}
\mathcal{L} b^{p q} & =\alpha K^{\alpha} b^{i j} \nabla_{i}\left(-b^{p r} b^{q s} \nabla_{j} h_{r s}\right) \\
& =2 \alpha K^{\alpha} b^{i j} b^{p k} b^{r m} b^{q s} \nabla_{i} h_{k m} \nabla_{j} h_{r s}-b^{p r} b^{q s} \mathcal{L} h_{r s} .
\end{aligned}
$$

Applying (5), we conclude that

$$
\begin{gathered}
\mathcal{L} b^{p q}=\alpha^{2} K^{\alpha} b^{p r} b^{q s} b^{i j} b^{k m} \nabla_{r} h_{i j} \nabla_{s} h_{k m}+\alpha K^{\alpha} b^{p r} b^{q s} b^{i j} b^{k m} \nabla_{r} h_{i k} \nabla_{s} h_{j m} \\
+\left\langle F, F^{k}\right\rangle \nabla_{k} b^{p q}-b^{p q}-(n \alpha-1) g^{p q} K^{\alpha}+\alpha K^{\alpha} H b^{p q} .
\end{gathered}
$$

Since $\nabla K^{\alpha}=\alpha K^{\alpha} b^{i j} \nabla h_{i j}$, the identity (6) follows.

\section{Classification of self-similar solutions: the case $\alpha \in\left[1 /(n+2), \frac{1}{2}\right]$}

In this section, we consider the case $\alpha \in\left[1 /(n+2), \frac{1}{2}\right]$. We begin with an algebraic lemma. 
LEMma 3. Assume $\alpha \in\left[1 /(n+2), \frac{1}{2}\right], \lambda_{1}, \ldots, \lambda_{n}$ are positive real numbers (not necessarily arranged in increasing order), and $\sigma_{1}, \ldots, \sigma_{n}$ are arbitrary real numbers. Then

$$
\begin{aligned}
Q:= & \sum_{i=1}^{n} \sigma_{i}^{2}+2 \sum_{i=1}^{n-1} \lambda_{n} \lambda_{i}^{-1} \sigma_{i}^{2} \\
& -4 \alpha \lambda_{n}\left(\sum_{i=1}^{n} \lambda_{i}^{-1} \sigma_{i}+\left((n \alpha-1) \lambda_{n}^{-1}-\alpha \sum_{i=1}^{n} \lambda_{i}^{-1}\right) \sum_{i=1}^{n} \sigma_{i}\right)\left(\sum_{i=1}^{n} \sigma_{i}\right) \\
& -2 \alpha^{2} \lambda_{n}\left(\sum_{i=1}^{n} \lambda_{i}^{-1}\right)\left(\sum_{i=1}^{n} \sigma_{i}\right)^{2}+\left(2 n \alpha^{2}+(n-1) \alpha-1\right)\left(\sum_{i=1}^{n} \sigma_{i}\right)^{2} \geqslant 0 .
\end{aligned}
$$

Moreover, if equality holds, then we either have $\sigma_{1}=\ldots=\sigma_{n}=0$, or we have $\alpha=1 /(n+2)$ and $\sigma_{1}=\ldots=\sigma_{n-1}=\frac{1}{3} \sigma_{n}$.

Proof. If $\sum_{i=1}^{n} \sigma_{i}=0$, the assertion is trivial. Hence, it suffices to consider the case $\sum_{i=1}^{n} \sigma_{i} \neq 0$. By scaling, we may assume $\sum_{i=1}^{n} \sigma_{i}=1$. Let us define

$$
\tau_{i}= \begin{cases}\sigma_{i}-\alpha, & \text { for } i=1, \ldots, n-1, \\ \sigma_{n}-1+(n-1) \alpha, & \text { for } i=n .\end{cases}
$$

Then

$$
\sum_{i=1}^{n} \tau_{i}=\sum_{i=1}^{n} \sigma_{i}-1=0
$$

and

Therefore, the quantity $Q$ satisfies

$$
\sum_{i=1}^{n} \lambda_{i}^{-1} \tau_{i}=\sum_{i=1}^{n} \lambda_{i}^{-1} \sigma_{i}+(n \alpha-1) \lambda_{n}^{-1}-\alpha \sum_{i=1}^{n} \lambda_{i}^{-1}
$$

$$
\begin{aligned}
Q= & \sum_{i=1}^{n-1}\left(\tau_{i}+\alpha\right)^{2}+\left(\tau_{n}+1-(n-1) \alpha\right)^{2}+2 \sum_{i=1}^{n-1} \lambda_{n} \lambda_{i}^{-1}\left(\tau_{i}+\alpha\right)^{2} \\
& \quad-4 \alpha \sum_{i=1}^{n} \lambda_{n} \lambda_{i}^{-1} \tau_{i}-2 \alpha^{2} \sum_{i=1}^{n} \lambda_{n} \lambda_{i}^{-1}+2 n \alpha^{2}+(n-1) \alpha-1 \\
= & \sum_{i=1}^{n} \tau_{i}^{2}+2 \alpha \sum_{i=1}^{n-1} \tau_{i}+2(1-(n-1) \alpha) \tau_{n}+(n-1) \alpha^{2}+(1-(n-1) \alpha)^{2} \\
& +2 \sum_{i=1}^{n-1} \lambda_{n} \lambda_{i}^{-1}\left(\tau_{i}^{2}+2 \alpha \tau_{i}+\alpha^{2}\right)-4 \alpha \sum_{i=1}^{n-1} \lambda_{n} \lambda_{i}^{-1} \tau_{i}-4 \alpha \tau_{n} \\
& \quad-2 \alpha^{2} \sum_{i=1}^{n-1} \lambda_{n} \lambda_{i}^{-1}-2 \alpha^{2}+2 n \alpha^{2}+(n-1) \alpha-1 \\
= & \sum_{i=1}^{n} \tau_{i}^{2}+2 \alpha \sum_{i=1}^{n-1} \tau_{i}+2(1-(n+1) \alpha) \tau_{n}+2 \sum_{i=1}^{n-1} \lambda_{n} \lambda_{i}^{-1} \tau_{i}^{2}+(n-1) \alpha((n+2) \alpha-1) .
\end{aligned}
$$


Using the identity $\sum_{i=1}^{n} \tau_{i}=0$, we obtain

$$
Q=\sum_{i=1}^{n} \tau_{i}^{2}+2(1-(n+2) \alpha) \tau_{n}+2 \sum_{i=1}^{n-1} \lambda_{n} \lambda_{i}^{-1} \tau_{i}^{2}+(n-1) \alpha((n+2) \alpha-1) .
$$

Moreover, the identity $\sum_{i=1}^{n} \tau_{i}=0$ gives

$$
\sum_{i=1}^{n} \tau_{i}^{2}=\sum_{i=1}^{n-1}\left(\tau_{i}+\frac{1}{n-1} \tau_{n}\right)^{2}-\frac{2}{n-1} \tau_{n} \sum_{i=1}^{n-1} \tau_{i}+\frac{n-2}{n-1} \tau_{n}^{2}=\sum_{i=1}^{n-1}\left(\tau_{i}+\frac{1}{n-1} \tau_{n}\right)^{2}+\frac{n}{n-1} \tau_{n}^{2} .
$$

Thus,

$$
\begin{gathered}
Q=\frac{n}{n-1}\left(\tau_{n}+\frac{n-1}{n}(1-(n+2) \alpha)\right)^{2}+\sum_{i=1}^{n-1}\left(\tau_{i}+\frac{1}{n-1} \tau_{n}\right)^{2} \\
+2 \sum_{i=1}^{n-1} \lambda_{n} \lambda_{i}^{-1} \tau_{i}^{2}+\frac{n-1}{n}(1-2 \alpha)((n+2) \alpha-1) .
\end{gathered}
$$

The right-hand side is clearly non-negative. Also, if equality holds, then $\tau_{1}=\ldots=\tau_{n}=0$ and $\alpha=1 /(n+2)$. This proves the lemma.

TheOrem 4. Assume $\alpha \in\left[1 /(n+2), \frac{1}{2}\right]$ and $\Sigma$ is a strictly convex closed smooth solution of $\left(*_{\alpha}\right)$. Then, either $\Sigma$ is a round sphere, or $\alpha=1 /(n+2)$ and $\Sigma$ is an ellipsoid.

Proof. Taking the trace in equation (6) gives

$$
\begin{aligned}
\mathcal{L} \operatorname{tr}(b)= & K^{-\alpha} b^{p r} b_{p}^{s} \nabla_{r} K^{\alpha} \nabla_{s} K^{\alpha}+\alpha K^{\alpha} b^{p r} b_{p}^{s} b^{i j} b^{k m} \nabla_{r} h_{i k} \nabla_{s} h_{j m} \\
& +\left\langle F, F_{i}\right\rangle \nabla^{i} \operatorname{tr}(b)-\operatorname{tr}(b)-n(n \alpha-1) K^{\alpha}+\alpha K^{\alpha} H \operatorname{tr}(b) .
\end{aligned}
$$

Using equation (4), we obtain

$$
\begin{aligned}
\mathcal{L}\left(K^{\alpha} \operatorname{tr}(b)\right)= & K^{\alpha} \mathcal{L} \operatorname{tr}(b)+\mathcal{L} K^{\alpha} \operatorname{tr}(b)+2 \alpha K^{\alpha} b^{i j} \nabla_{i} K^{\alpha} \nabla_{j} \operatorname{tr}(b) \\
= & b^{p r} b_{p}^{s} \nabla_{r} K^{\alpha} \nabla_{s} K^{\alpha}+\alpha K^{2 \alpha} b^{p r} b_{p}^{s} b^{i j} b^{k m} \nabla_{r} h_{i k} \nabla_{s} h_{j m} \\
& +\left\langle F, F_{i}\right\rangle \nabla^{i}\left(K^{\alpha} \operatorname{tr}(b)\right)+(n \alpha-1) K^{\alpha} \operatorname{tr}(b)-n(n \alpha-1) K^{2 \alpha} \\
& +2 \alpha K^{\alpha} b^{i j} \nabla_{i} K^{\alpha} \nabla_{j} \operatorname{tr}(b) .
\end{aligned}
$$

Using (2), it follows that the function

$$
Z=K^{\alpha} \operatorname{tr}(b)-\frac{n \alpha-1}{2 \alpha}|F|^{2}
$$

satisfies

$$
\begin{aligned}
\mathcal{L} Z=b^{p r} b_{p}^{s} \nabla_{r} K^{\alpha} \nabla_{s} K^{\alpha}+\alpha K^{2 \alpha} b^{p r} b_{p}^{s} b^{i j} b^{k m} \nabla_{r} h_{i k} \nabla_{s} h_{j m} \\
+\left\langle F, F_{i}\right\rangle \nabla^{i}\left(K^{\alpha} \operatorname{tr}(b)\right)+2 \alpha K^{\alpha} b^{i j} \nabla_{i} K^{\alpha} \nabla_{j} \operatorname{tr}(b) .
\end{aligned}
$$


Using (3), we obtain

$$
\frac{1}{2} \nabla^{i}|F|^{2}=\left\langle F, F^{i}\right\rangle=b^{i j} \nabla_{j} K^{\alpha}
$$

and hence

$$
\nabla_{i} Z=K^{\alpha} \nabla_{i} \operatorname{tr}(b)+\operatorname{tr}(b) \nabla_{i} K^{\alpha}-\frac{n \alpha-1}{\alpha} b_{i}^{j} \nabla_{j} K^{\alpha}
$$

This gives

$$
\left\langle F, F_{i}\right\rangle \nabla^{i}\left(K^{\alpha} \operatorname{tr}(b)\right)=b^{i j} \nabla_{i} K^{\alpha} \nabla_{j} Z+\frac{n \alpha-1}{\alpha} b^{i k} b_{k}^{j} \nabla_{i} K^{\alpha} \nabla_{j} K^{\alpha}
$$

and

$$
2 \alpha K^{\alpha} b^{i j} \nabla_{i} K^{\alpha} \nabla_{j} \operatorname{tr}(b)=2 \alpha b^{i j} \nabla_{i} K^{\alpha} \nabla_{j} Z-\left(2 \alpha b^{i j} \operatorname{tr}(b)-2(n \alpha-1) b^{i k} b_{k}^{j}\right) \nabla_{i} K^{\alpha} \nabla_{j} K^{\alpha} .
$$

Substituting these identities into (8) gives

$$
\begin{aligned}
\mathcal{L} Z+(2 \alpha-1) b^{i j} \nabla_{i} K^{\alpha} \nabla_{j} Z & \\
=4 & \alpha b^{i j} \nabla_{i} K^{\alpha} \nabla_{j} Z+\alpha K^{2 \alpha} b^{p r} b_{p}^{s} b^{i j} b^{k m} \nabla_{r} h_{i k} \nabla_{s} h_{j m} \\
& \quad+\left(-2 \alpha b^{i j} \operatorname{tr}(b)+\left(2 n \alpha+n-1-\alpha^{-1}\right) b^{i k} b_{k}^{j}\right) \nabla_{i} K^{\alpha} \nabla_{j} K^{\alpha} .
\end{aligned}
$$

Let us fix an arbitrary point $p$. We can choose an orthonormal frame so that $h_{i j}(p)=\lambda_{i} \delta_{i j}$. With this understood, we have

$$
\nabla_{i} K^{\alpha}=\alpha K^{\alpha} \sum_{j=1}^{n} \lambda_{j}^{-1} \nabla_{i} h_{j j} \quad \text { and } \quad \nabla_{i} \operatorname{tr}(b)=-\sum_{j=1}^{n} \lambda_{j}^{-2} \nabla_{i} h_{j j} .
$$

Let $D$ denote the set of all triplets $(i, j, k)$ such that $i, j$ and $k$ are pairwise distinct. Then, by using (9) and (10), we have

$$
\begin{aligned}
& \alpha^{-1} K^{-2 \alpha}\left(\mathcal{L} Z+(2 \alpha-1) b^{i j} \nabla_{i} K^{\alpha} \nabla_{j} Z\right) \\
& =\sum_{D} \lambda_{i}^{-2} \lambda_{j}^{-1} \lambda_{k}^{-1}\left(\nabla_{i} h_{j k}\right)^{2}+4 \alpha \sum_{k} \lambda_{k}^{-1}\left(\nabla_{k} \log K\right)\left(K^{-\alpha} \nabla_{k} Z\right) \\
& \quad+\sum_{k} \sum_{i} \lambda_{k}^{-2} \lambda_{i}^{-2}\left(\nabla_{k} h_{i i}\right)^{2}+2 \sum_{k} \sum_{i \neq k} \lambda_{k}^{-1} \lambda_{i}^{-3}\left(\nabla_{k} h_{i i}\right)^{2} \\
& \quad+\sum_{k} \lambda_{k}^{-1}\left(-2 \alpha^{2} \operatorname{tr}(b)+\left(2 n \alpha^{2}+(n-1) \alpha-1\right) \lambda_{k}^{-1}\right)\left(\nabla_{k} \log K\right)^{2} .
\end{aligned}
$$

We claim that, for each $k$, the following holds:

$$
\begin{array}{r}
\sum_{i} \lambda_{k}^{-1} \lambda_{i}^{-2}\left(\nabla_{k} h_{i i}\right)^{2}+2 \sum_{i \neq k} \lambda_{i}^{-3}\left(\nabla_{k} h_{i i}\right)^{2}+4 \alpha\left(\nabla_{k} \log K\right)\left(K^{-\alpha} \nabla_{k} Z\right) \\
+\left(-2 \alpha^{2} \operatorname{tr}(b)+\left(2 n \alpha^{2}+(n-1) \alpha-1\right) \lambda_{k}^{-1}\right)\left(\nabla_{k} \log K\right)^{2} \geqslant 0 .
\end{array}
$$


Notice that

$$
K^{-\alpha} \nabla_{k} Z=-\sum_{i} \lambda_{i}^{-2} \nabla_{k} h_{i i}+\left(\alpha \operatorname{tr}(b)-(n \alpha-1) \lambda_{k}^{-1}\right) \nabla_{k} \log K
$$

After relabeling indices, we may assume $k=n$. If we put $\sigma_{i}:=\lambda_{i}^{-1} \nabla_{n} h_{i i}$, then the assertion follows from Lemma 3. This proves (12). Combining (11) and (12), we conclude that

$$
\mathcal{L} Z+(2 \alpha-1) b^{i j} \nabla_{i} K^{\alpha} \nabla_{j} Z \geqslant 0
$$

at each point $p$. Therefore, by the strong maximum principle, $Z$ is a constant. Hence, the left-hand side of (11) is zero. Therefore, $\nabla_{i} h_{j k}=0$ if $i, j$ and $k$ are all distinct. Moreover, since we have equality in the lemma, we either have $\lambda_{i}^{-1} \nabla_{k} h_{i i}=0$ for all $i$ and $k$, or we have $\alpha=1 /(n+2)$ and $\lambda_{i}^{-1} \nabla_{k} h_{i i}=\frac{1}{3} \lambda_{k}^{-1} \nabla_{k} h_{k k}$ for $i \neq k$.

In the first case, we conclude that $\nabla_{i} h_{j k}=0$ for all $i, j$ and $k$, and thus $\Sigma$ is a round sphere.

In the second case, we obtain

$$
\lambda_{i}^{-1} \nabla_{k} h_{i i}=\frac{1}{n+2} \nabla_{k} \log K \text { for } i \neq k \quad \text { and } \quad \lambda_{k}^{-1} \nabla_{k} h_{k k}=\frac{3}{n+2} \nabla_{k} \log K
$$

This gives

$C_{i j k}=\frac{1}{2} K^{-1 /(n+2)} \nabla_{k} h_{i j}+\frac{1}{2} h_{j k} \nabla_{i} K^{-1 /(n+2)}+\frac{1}{2} h_{k i} \nabla_{j} K^{-1 /(n+2)}+\frac{1}{2} h_{i j} \nabla_{k} K^{-1 /(n+2)}=0$

for all $i, j$ and $k$. Since the cubic form $C_{i j k}$ vanishes everywhere, the surface is an ellipsoid by the Berwald-Pick theorem (see e.g. [19, Theorem 4.5]). This proves the theorem.

\section{Classification of self-similar solutions: the case $\alpha \in\left(\frac{1}{2}, \infty\right)$}

We now turn to the case $\alpha \in\left(\frac{1}{2}, \infty\right)$. In the following, we denote by $\lambda_{1} \leqslant \ldots \leqslant \lambda_{n}$ the eigenvalues of the second fundamental form, arranged in increasing order. Each eigenvalue defines a Lipschitz continuous function on $M$.

LEMMA 5. Suppose that $\varphi$ is a smooth function such that $\lambda_{1} \geqslant \varphi$ everywhere and $\lambda_{1}=\varphi$ at $\bar{p}$. Let $\mu$ denote the multiplicity of the smallest curvature eigenvalue at $\bar{p}$, so that $\lambda_{1}=\ldots=\lambda_{\mu}<\lambda_{\mu+1} \leqslant \ldots \leqslant \lambda_{n}$. Then, at $\bar{p}, \nabla_{i} h_{k l}=\nabla_{i} \varphi \delta_{k l}$ for $1 \leqslant k, l \leqslant \mu$. Moreover,

$$
\nabla_{i} \nabla_{i} \varphi \leqslant \nabla_{i} \nabla_{i} h_{11}-2 \sum_{l>\mu}\left(\lambda_{l}-\lambda_{1}\right)^{-1}\left(\nabla_{i} h_{1 l}\right)^{2} .
$$

at $\bar{p}$. 
Proof. Fix an index $i$, and let $\gamma(s)$ be the geodesic satisfying $\gamma(0)=\bar{p}$ and $\gamma^{\prime}(0)=e_{i}$. Moreover, let $v(s)$ be a vector field along $\gamma$ such that $v(0) \in \operatorname{span}\left\{e_{1}, \ldots, e_{\mu}\right\}$ and $v^{\prime}(0) \in$ $\operatorname{span}\left\{e_{\mu+1}, \ldots, e_{n}\right\}$. Then, the function $s \mapsto h(v(s), v(s))-\varphi(\gamma(s))|v(s)|^{2}$ has a local minimum at $s=0$. This gives

$$
\begin{aligned}
0 & =\left.\frac{d}{d s}\left(h(v(s), v(s))-\varphi(\gamma(s))|v(s)|^{2}\right)\right|_{s=0} \\
& =\nabla_{i} h(v(0), v(0))+2 h\left(v(0), v^{\prime}(0)\right)-\nabla_{i} \varphi|v(0)|^{2}-2\left\langle v(0), v^{\prime}(0)\right\rangle \\
& =\nabla_{i} h(v(0), v(0))-\nabla_{i} \varphi|v(0)|^{2}
\end{aligned}
$$

Since $v(0) \in \operatorname{span}\left\{e_{1}, \ldots, e_{\mu}\right\}$ is arbitrary, it follows that $\nabla_{i} h_{k l}=\nabla_{i} \varphi \delta_{k l}$ for $1 \leqslant k, l \leqslant \mu$ at the point $\bar{p}$.

We next consider the second derivative. To that end, we choose $v(0)=e_{1}$,

$$
v^{\prime}(0)=-\sum_{l>\mu}\left(\lambda_{l}-\lambda_{1}\right)^{-1} \nabla_{i} h_{1 l} e_{l},
$$

and $v^{\prime \prime}(0)=0$. Since the function $s \mapsto h(v(s), v(s))-\varphi(\gamma(s))|v(s)|^{2}$ has a local minimum at $s=0$, we obtain

$$
\begin{aligned}
& 0 \leqslant\left.\frac{d^{2}}{d s^{2}}\left(h(v(s), v(s))-\varphi(\gamma(s))|v(s)|^{2}\right)\right|_{s=0} \\
& =\nabla_{i} \nabla_{i} h(v(0), v(0))+4 \nabla_{i} h\left(v(0), v^{\prime}(0)\right)+2 h\left(v^{\prime}(0), v^{\prime}(0)\right) \\
& \quad \quad-\nabla_{i} \nabla_{i} \varphi|v(0)|^{2}-4 \nabla_{i} \varphi\left\langle v(0), v^{\prime}(0)\right\rangle-2 \varphi\left|v^{\prime}(0)\right|^{2} \\
& =\nabla_{i} \nabla_{i} h_{11}-4 \sum_{l>\mu}\left(\lambda_{l}-\lambda_{1}\right)^{-1}\left(\nabla_{i} h_{1 l}\right)^{2}+2 \sum_{l>\mu} \lambda_{l}\left(\lambda_{l}-\lambda_{1}\right)^{-2}\left(\nabla_{i} h_{1 l}\right)^{2} \\
& \quad-\nabla_{i} \nabla_{i} \varphi-2 \sum_{l>\mu} \lambda_{1}\left(\lambda_{l}-\lambda_{1}\right)^{-2}\left(\nabla_{i} h_{1 l}\right)^{2} \\
& =\nabla_{i} \nabla_{i} h_{11}-2 \sum_{l>\mu}\left(\lambda_{l}-\lambda_{1}\right)^{-2}\left(\nabla_{i} h_{1 l}\right)^{2}-\nabla_{i} \nabla_{i} \varphi .
\end{aligned}
$$

This proves the assertion.

TheOREM 6. Assume $\alpha \in\left(\frac{1}{2}, \infty\right)$ and $\Sigma$ is a strictly convex closed smooth solution of $\left(*_{\alpha}\right)$. Then $\Sigma$ is a round sphere.

Proof. Let us consider the function

$$
W=K^{\alpha} \lambda_{1}^{-1}-\frac{n \alpha-1}{2 n \alpha}|F|^{2} .
$$


Let us consider an arbitrary point $\bar{p}$ where $W$ attains its maximum. As above, we denote by $\mu$ the multiplicity of the smallest eigenvalue of the second fundamental form. Let us define a smooth function $\varphi$ such that

$$
W(\bar{p})=K^{\alpha} \varphi^{-1}-\frac{n \alpha-1}{2 n \alpha}|F|^{2}
$$

Since $W$ attains its maximum at $\bar{p}$, we have $\lambda_{1} \geqslant \varphi$ everywhere and $\lambda_{1}=\varphi$ at $\bar{p}$. Therefore, we may apply the previous lemma. Hence, at the point $\bar{p}$, we have $\nabla_{i} h_{k l}=\nabla_{i} \varphi \delta_{k l}$ for $1 \leqslant k, l \leqslant \mu$. Moreover,

$$
\nabla_{k} \nabla_{k} \varphi \leqslant \nabla_{k} \nabla_{k} h_{11}-2 \sum_{l>\mu}\left(\lambda_{l}-\lambda_{1}\right)^{-1}\left(\nabla_{k} h_{1 l}\right)^{2}
$$

at $\bar{p}$. We multiply both sides by $\alpha K^{\alpha} \lambda_{k}^{-1}$ and sum over $k$. This gives

$$
\mathcal{L} \varphi \leqslant \mathcal{L} h_{11}-2 \alpha K^{\alpha} \sum_{k} \sum_{l>\mu} \lambda_{k}^{-1}\left(\lambda_{l}-\lambda_{1}\right)^{-1}\left(\nabla_{k} h_{1 l}\right)^{2}
$$

By (5), we have

$$
\begin{aligned}
\mathcal{L} h_{i j}=- & K^{-\alpha} \nabla_{i} K^{\alpha} \nabla_{j} K^{\alpha}+\alpha K^{\alpha} b^{p r} b^{q s} \nabla_{i} h_{r s} \nabla_{j} h_{p q} \\
& +\left\langle F, F_{k}\right\rangle \nabla^{k} h_{i j}+h_{i j}+(n \alpha-1) h_{i k} h_{j}^{k} K^{\alpha}-\alpha K^{\alpha} H h_{i j} .
\end{aligned}
$$

Thus,

$$
\begin{aligned}
\mathcal{L} \varphi \leqslant- & 2 \alpha K^{\alpha} \sum_{k} \sum_{l>\mu} \lambda_{k}^{-1}\left(\lambda_{l}-\lambda_{1}\right)^{-1}\left(\nabla_{k} h_{1 l}\right)^{2}-K^{-\alpha}\left(\nabla_{1} K^{\alpha}\right)^{2}+\alpha K^{\alpha} \sum_{k, l} \lambda_{k}^{-1} \lambda_{l}^{-1}\left(\nabla_{k} h_{1 l}\right)^{2} \\
& +\sum_{k}\left\langle F, F_{k}\right\rangle \nabla_{k} h_{11}+\lambda_{1}+(n \alpha-1) \lambda_{1}^{2} K^{\alpha}-\alpha K^{\alpha} H \lambda_{1} .
\end{aligned}
$$

Using the estimate

$$
\begin{aligned}
-2 \alpha K^{\alpha} & \sum_{k} \sum_{l>\mu} \lambda_{k}^{-1}\left(\lambda_{l}-\lambda_{1}\right)^{-1}\left(\nabla_{k} h_{1 l}\right)^{2}+\alpha K^{\alpha} \sum_{k, l} \lambda_{k}^{-1} \lambda_{l}^{-1}\left(\nabla_{k} h_{1 l}\right)^{2} \\
& =-\alpha K^{\alpha} \sum_{k} \sum_{l>\mu} \lambda_{k}^{-1}\left(2\left(\lambda_{l}-\lambda_{1}\right)^{-1}-\lambda_{l}^{-1}\right)\left(\nabla_{k} h_{1 l}\right)^{2}+\alpha K^{\alpha} \sum_{k} \lambda_{k}^{-1} \lambda_{1}^{-1}\left(\nabla_{k} h_{11}\right)^{2} \\
& \leqslant-\alpha K^{\alpha} \sum_{l>\mu} \lambda_{1}^{-1}\left(2\left(\lambda_{l}-\lambda_{1}\right)^{-1}-\lambda_{l}^{-1}\right)\left(\nabla_{1} h_{1 l}\right)^{2}+\alpha K^{\alpha} \sum_{k} \lambda_{k}^{-1} \lambda_{1}^{-1}\left(\nabla_{k} h_{11}\right)^{2} \\
& =-2 \alpha K^{\alpha} \sum_{k>\mu} \lambda_{1}^{-1}\left(\left(\lambda_{k}-\lambda_{1}\right)^{-1}-\lambda_{k}^{-1}\right)\left(\nabla_{k} h_{11}\right)^{2}+\alpha K^{\alpha} \lambda_{1}^{-2}\left(\nabla_{1} h_{11}\right)^{2},
\end{aligned}
$$


we obtain

$$
\begin{aligned}
\mathcal{L} \varphi \leqslant & -2 \alpha K^{\alpha} \lambda_{1}^{-1} \sum_{k>\mu}\left(\left(\lambda_{k}-\lambda_{1}\right)^{-1}-\lambda_{k}^{-1}\right)\left(\nabla_{k} h_{11}\right)^{2}+\alpha K^{\alpha} \lambda_{1}^{-2}\left(\nabla_{1} h_{11}\right)^{2}-K^{-\alpha}\left(\nabla_{1} K^{\alpha}\right)^{2} \\
& +\sum_{k}\left\langle F, F_{k}\right\rangle \nabla_{k} h_{11}+\lambda_{1}+(n \alpha-1) \lambda_{1}^{2} K^{\alpha}-\alpha K^{\alpha} H \lambda_{1} .
\end{aligned}
$$

Since $\nabla_{k} \varphi=\nabla_{k} h_{11}$, it follows that

$$
\begin{aligned}
\mathcal{L}\left(\varphi^{-1}\right) \geqslant 2 & \alpha K^{\alpha} \lambda_{1}^{-3} \sum_{k} \lambda_{k}^{-1}\left(\nabla_{k} h_{11}\right)^{2} \\
& +2 \alpha K^{\alpha} \lambda_{1}^{-3} \sum_{k>\mu}\left(\left(\lambda_{k}-\lambda_{1}\right)^{-1}-\lambda_{k}^{-1}\right)\left(\nabla_{k} h_{11}\right)^{2} \\
& -\alpha K^{\alpha} \lambda_{1}^{-4}\left(\nabla_{1} h_{11}\right)^{2}+K^{-\alpha} \lambda_{1}^{-2}\left(\nabla_{1} K^{\alpha}\right)^{2} \\
& +\sum_{k}\left\langle F, F_{k}\right\rangle \nabla_{k}\left(\lambda_{1}^{-1}\right)-\lambda_{1}^{-1}-(n \alpha-1) K^{\alpha}+\alpha K^{\alpha} H \lambda_{1}^{-1} \\
=2 \alpha & K^{\alpha} \lambda_{1}^{-3} \sum_{k>\mu}\left(\lambda_{k}-\lambda_{1}\right)^{-1}\left(\nabla_{k} h_{11}\right)^{2} \\
& +\alpha K^{\alpha} \lambda_{1}^{-4}\left(\nabla_{1} h_{11}\right)^{2}+K^{-\alpha} \lambda_{1}^{-2}\left(\nabla_{1} K^{\alpha}\right)^{2} \\
& +\sum_{k}\left\langle F, F_{k}\right\rangle \nabla_{k}\left(\lambda_{1}^{-1}\right)-\lambda_{1}^{-1}-(n \alpha-1) K^{\alpha}+\alpha K^{\alpha} H \lambda_{1}^{-1} .
\end{aligned}
$$

This gives

$$
\begin{aligned}
\mathcal{L}\left(K^{\alpha} \varphi^{-1}\right)= & K^{\alpha} \mathcal{L}\left(\varphi^{-1}\right)+\varphi^{-1} \mathcal{L}\left(K^{\alpha}\right)+2 \alpha K^{\alpha} \sum_{k} \lambda_{k}^{-1} \nabla_{k} K^{\alpha} \nabla_{k}\left(\varphi^{-1}\right) \\
\geqslant & 2 \alpha \sum_{k} \lambda_{k}^{-1} \nabla_{k} K^{\alpha} \nabla_{k}\left(K^{\alpha} \varphi^{-1}\right)-2 \alpha \lambda_{1}^{-1} \sum_{k} \lambda_{k}^{-1}\left(\nabla_{k} K^{\alpha}\right)^{2} \\
& +2 \alpha K^{2 \alpha} \lambda_{1}^{-3} \sum_{k>\mu}\left(\lambda_{k}-\lambda_{1}\right)^{-1}\left(\nabla_{k} h_{11}\right)^{2} \\
& +\alpha K^{2 \alpha} \lambda_{1}^{-4}\left(\nabla_{1} h_{11}\right)^{2}+\lambda_{1}^{-2}\left(\nabla_{1} K^{\alpha}\right)^{2} \\
& +\sum_{k}\left\langle F, F_{k}\right\rangle \nabla_{k}\left(K^{\alpha} \varphi^{-1}\right)+(n \alpha-1) K^{\alpha} \lambda_{1}^{-1}-(n \alpha-1) K^{2 \alpha}
\end{aligned}
$$

By assumption, the function

$$
K^{\alpha} \varphi^{-1}-\frac{n \alpha-1}{2 n \alpha}|F|^{2}
$$

is constant. Consequently,

$$
\nabla_{k}\left(K^{\alpha} \varphi^{-1}\right)=\frac{n \alpha-1}{2 n \alpha} \nabla_{k}|F|^{2},
$$


and

$$
\begin{aligned}
0= & \mathcal{L}\left(K^{\alpha} \varphi^{-1}-\frac{n \alpha-1}{2 n \alpha}|F|^{2}\right) \\
\geqslant & \frac{n \alpha-1}{n} \sum_{k} \lambda_{k}^{-1} \nabla_{k} K^{\alpha} \nabla_{k}|F|^{2}-2 \alpha \lambda_{1}^{-1} \sum_{k} \lambda_{k}^{-1}\left(\nabla_{k} K^{\alpha}\right)^{2} \\
& +2 \alpha K^{2 \alpha} \lambda_{1}^{-3} \sum_{k>\mu}\left(\lambda_{k}-\lambda_{1}\right)^{-1}\left(\nabla_{k} h_{11}\right)^{2} \\
& +\alpha K^{2 \alpha} \lambda_{1}^{-4}\left(\nabla_{1} h_{11}\right)^{2}+\lambda_{1}^{-2}\left(\nabla_{1} K^{\alpha}\right)^{2} \\
& +\frac{n \alpha-1}{2 n \alpha} \sum_{k}\left\langle F, F_{k}\right\rangle \nabla_{k}|F|^{2}+(n \alpha-1) K^{\alpha}\left(\lambda_{1}^{-1}-\frac{1}{n} \operatorname{tr}(b)\right) .
\end{aligned}
$$

Recall that

$$
\frac{1}{2} \nabla_{k}|F|^{2}=\left\langle F, F_{k}\right\rangle=\lambda_{k}^{-1} \nabla_{k} K^{\alpha}
$$

by (3). Moreover, using the identity $\nabla_{k} \varphi=\nabla_{k} h_{11}$, we obtain

$$
0=\nabla_{k}\left(K^{\alpha} \varphi^{-1}\right)-\frac{n \alpha-1}{2 n \alpha} \nabla_{k}|F|^{2}=\left(\lambda_{1}^{-1}-\frac{n \alpha-1}{n \alpha} \lambda_{k}^{-1}\right) \nabla_{k} K^{\alpha}-K^{\alpha} \lambda_{1}^{-2} \nabla_{k} h_{11}
$$

at $\bar{p}$. Note that, if $2 \leqslant l \leqslant \mu$, then $\nabla_{k} h_{1 l}=0$ for all $k$. Putting $k=1$ gives $\nabla_{l} h_{11}=0$ and $\nabla_{l} K=0$ for $2 \leqslant l \leqslant \mu$. Putting these facts together, we obtain

$$
\begin{aligned}
& 0 \geqslant \frac{2(n \alpha-1)}{n} \sum_{k} \lambda_{k}^{-2}\left(\nabla_{k} K^{\alpha}\right)^{2}-2 \alpha \lambda_{1}^{-1} \sum_{k} \lambda_{k}^{-1}\left(\nabla_{k} K^{\alpha}\right)^{2} \\
& +2 \alpha \lambda_{1} \sum_{k>\mu}\left(\lambda_{k}-\lambda_{1}\right)^{-1}\left(\lambda_{1}^{-1}-\frac{n \alpha-1}{n \alpha} \lambda_{k}^{-1}\right)^{2}\left(\nabla_{k} K^{\alpha}\right)^{2} \\
& +\frac{1}{n^{2} \alpha} \lambda_{1}^{-2}\left(\nabla_{1} K^{\alpha}\right)^{2}+\lambda_{1}^{-2}\left(\nabla_{1} K^{\alpha}\right)^{2} \\
& +\frac{n \alpha-1}{n \alpha} \sum_{k} \lambda_{k}^{-2}\left(\nabla_{k} K^{\alpha}\right)^{2}+(n \alpha-1) K^{\alpha}\left(\lambda_{1}^{-1}-\frac{1}{n} \operatorname{tr}(b)\right) \text {. }
\end{aligned}
$$

Using the identities

$$
\begin{gathered}
\frac{2(n \alpha-1)}{n} \lambda_{k}^{-2}-2 \alpha \lambda_{1}^{-1} \lambda_{k}^{-1}+ \\
=2 \alpha \lambda_{1}\left(\lambda_{k}-\lambda_{1}\right)^{-1}\left(\lambda_{1}^{-1}-\frac{n \alpha-1}{n \alpha} \lambda_{k}^{-1}\right)^{2}+\frac{n \alpha-1}{n \alpha} \lambda_{k}^{-2} \\
=\left(\frac{n \alpha-1}{n \alpha}+\frac{2}{n}+\frac{2}{n^{2} \alpha} \lambda_{1}\left(\lambda_{k}-\lambda_{1}\right)^{-1}\right) \lambda_{k}^{-2}
\end{gathered}
$$

and

$$
\frac{2(n \alpha-1)}{n} \lambda_{1}^{-2}-2 \alpha \lambda_{1}^{-2}+\frac{1}{n^{2} \alpha} \lambda_{1}^{-2}+\lambda_{1}^{-2}+\frac{n \alpha-1}{n \alpha} \lambda_{1}^{-2}=\frac{n-1}{n^{2} \alpha}(2 n \alpha-1) \lambda_{1}^{-2},
$$


the previous inequality can be rewritten as follows:

$$
\begin{aligned}
0 \geqslant \sum_{k>\mu} & \left(\frac{n \alpha-1}{n \alpha}+\frac{2}{n}+\frac{2}{n^{2} \alpha} \lambda_{1}\left(\lambda_{k}-\lambda_{1}\right)^{-1}\right) \lambda_{k}^{-2}\left(\nabla_{k} K^{\alpha}\right)^{2} \\
& +\frac{n-1}{n^{2} \alpha}(2 n \alpha-1) \lambda_{1}^{-2}\left(\nabla_{1} K^{\alpha}\right)^{2}+(n \alpha-1) K^{\alpha}\left(\lambda_{1}^{-1}-\frac{1}{n} \operatorname{tr}(b)\right) .
\end{aligned}
$$

Since $\alpha>1 / n$, it follows that $\bar{p}$ is an umbilical point. As $\bar{p}$ is an umbilical point and $\alpha>\frac{1}{2}$, there exists a neighborhood $U$ of $\bar{p}$ with the property that

$$
\begin{aligned}
& \alpha^{-1} K^{-2 \alpha}\left(\mathcal{L} Z-(2 \alpha+1) b^{i j} \nabla_{i} K^{\alpha} \nabla_{j} Z\right) \\
& =\sum_{D} \lambda_{i}^{-2} \lambda_{j}^{-1} \lambda_{k}^{-1}\left(\nabla_{i} h_{j k}\right)^{2} \\
& \quad+\sum_{k} \sum_{i} \lambda_{k}^{-2} \lambda_{i}^{-2}\left(\nabla_{k} h_{i i}\right)^{2}+2 \sum_{k} \sum_{i \neq k} \lambda_{k}^{-1} \lambda_{i}^{-3}\left(\nabla_{k} h_{i i}\right)^{2} \\
& \quad+\sum_{k} \lambda_{k}^{-1}\left(-2 \alpha^{2} \operatorname{tr}(b)+\left(2 n \alpha^{2}+(n-1) \alpha-1\right) \lambda_{k}^{-1}\right)\left(\nabla_{k} \log K\right)^{2} \geqslant 0
\end{aligned}
$$

at each point in $U$. (Indeed, if $n \geqslant 3$, the last inequality follows immediately from the fact that $(n-1) \alpha-1 \geqslant 0$. For $n=2$ the last inequality follows from a straightforward calculation.)

Now, since $\bar{p}$ is an umbilical point, we have $Z(p) \leqslant n W(p) \leqslant n W(\bar{p})=Z(\bar{p})$ for each point $p \in U$. Thus, $Z$ attains a local maximum at $\bar{p}$. By the strong maximum principle, $Z(p)=Z(\bar{p})$ for all points $p \in U$. This implies that $W(p)=W(\bar{p})$ for all points $p \in U$. Thus, the set of all points where $W$ attains its maximum is open. Consequently, $W$ is constant. This implies that $\Sigma$ is umbilical, and hence a round sphere.

\section{Proof of Theorem 1}

Suppose that we have any strictly convex solution to the flow with speed $-K^{\alpha} \nu$, where $\alpha \in[1 /(n+2), \infty)$. It is known that the flow converges to a soliton after rescaling; for $\alpha>1 /(n+2)$, this follows from [5, Theorem 6.2], while for $\alpha=1 /(n+2)$ this follows from results in $[3, \S 9]$. By Theorems 4 and 6 , either the limit is a round sphere, or $\alpha=1 /(n+2)$ and the limit is an ellipsoid. 


\section{References}

[1] Andrews, B., Contraction of convex hypersurfaces by their affine normal. J. Differential Geom., 43 (1996), 207-230.

[2] - Gauss curvature flow: the fate of the rolling stones. Invent. Math., 138 (1999), 151-161.

[3] - Motion of hypersurfaces by Gauss curvature. Pacific J. Math., 195 (2000), 1-34.

[4] Andrews, B. \& Chen, X., Surfaces moving by powers of Gauss curvature. Pure Appl. Math. Q., 8 (2012), 825-834.

[5] Andrews, B., Guan, P. \& Ni, L., Flow by powers of the Gauss curvature. Adv. Math., 299 (2016), 174-201.

[6] Bethuel, F., Huisken, G., Müller, S. \& Steffen, K., Calculus of Variations and Geometric Evolution Problems. Lecture Notes in Mathematics, 1713. Springer, BerlinHeidelberg, 1999.

[7] Calabi, E., Complete affine hyperspheres. I, in Symposia Mathematica, Vol. X (Convegno di Geometria Differenziale, INdAM, Rome, 1971), pp. 19-38. Academic Press, London, 1972.

[8] Choi, K., The Gauss Curvature Flow: Regularity and Asymptotic Behavior. Ph.D. Thesis, Columbia University, New York, NY, 2017.

[9] Chow, B., Deforming convex hypersurfaces by the $n$th root of the Gaussian curvature. $J$. Differential Geom., 22 (1985), 117-138.

[10] - On Harnack's inequality and entropy for the Gaussian curvature flow. Comm. Pure Appl. Math., 44 (1991), 469-483.

[11] Chow, B. \& Gulliver, R., Aleksandrov reflection and nonlinear evolution equations. I. The $n$-sphere and n-ball. Calc. Var. Partial Differential Equations, 4 (1996), 249-264.

[12] Chow, B. \& Hamilton, R. S., The cross curvature flow of 3-manifolds with negative sectional curvature. Turkish J. Math., 28 (2004), 1-10.

[13] Chow, B. \& Tsai, D. H., Nonhomogeneous Gauss curvature flows. Indiana Univ. Math. J., 47 (1998), 965-994.

[14] Firey, W. J., Shapes of worn stones. Mathematika, 21 (1974), 1-11.

[15] Gerhardt, C., Non-scale-invariant inverse curvature flows in Euclidean space. Calc. Var. Partial Differential Equations, 49 (2014), 471-489.

[16] Guan, P. \& Ni, L., Entropy and a convergence theorem for Gauss curvature flow in high dimension. J. Eur. Math. Soc. (JEMS), 19 (2017), 3735-3761.

[17] Hamilton, R. S., Remarks on the entropy and Harnack estimates for the Gauss curvature flow. Comm. Anal. Geom., 2 (1994), 155-165.

[18] Huisken, G., Flow by mean curvature of convex surfaces into spheres. J. Differential Geom., 20 (1984), 237-266.

[19] Nomizu, K. \& Sasaki, T., Affine Differential Geometry. Cambridge Tracts in Mathematics, 111. Cambridge University Press, Cambridge, 1994.

[20] Schnürer, O. C., Surfaces expanding by the inverse Gauß curvature flow. J. Reine Angew. Math., 600 (2006), 117-134.

[21] Tso, K., Deforming a hypersurface by its Gauss-Kronecker curvature. Comm. Pure Appl. Math., 38 (1985), 867-882. 
Simon Brendle

Department of Mathematics Columbia University

2990 Broadway

New York, NY 10027

U.S.A.

simon.brendle@columbia.edu

Panagiota Daskalopoulos

Department of Mathematics

Columbia University

2990 Broadway

New York, NY 10027

U.S.A.

pdaskalo@math.columbia.edu

Received December 6, 2016
Kyeongsu Choi

Department of Mathematics Columbia University

2990 Broadway

New York, NY 10027

U.S.A.

kschoi@math.columbia.edu 